\title{
Cardiotoxicity and Cardiac Monitoring Among Anthracycline-Treated Cancer Patients: A Retrospective Cohort Study
}

\author{
Hadeel Alkofide (D' \\ Lamya Alnaim (D) \\ Nora Alorf ${ }^{2}$ \\ Ward Alessa ${ }^{2}$ \\ Ghada Bawazeer' \\ 'Department of Clinical Pharmacy, \\ College of Pharmacy, King Saud \\ University, Riyadh, Saudi Arabia; ${ }^{2}$ College \\ of Pharmacy, King Saud University, \\ Riyadh, Saudi Arabia
}

Purpose: Cardiotoxicity is a common complication associated with anthracyclines. Little is known regarding the rate of anthracyclines-related acute and chronic cardiotoxicity and adherence to cardiac monitoring recommendations among cancer patients.

Patients and Methods: A single-centre retrospective cohort study was conducted from 2015 to 2018 on patients with cancer, 18 years of age and older, on anthracyclines without a history of cardiovascular diseases. Data on demographic information, comorbidities, cardiovascular events, monitoring parameters, and treatment details were obtained. The primary outcome was the incidence of anthracyclines-related cardiotoxicity both acute and chronic. The secondary outcome was to determine adherence to guideline recommendations for monitoring anthracyclines-related cardiotoxicity based on the American Society of Clinical Oncology clinical practice guidelines. Analyses included descriptive statistics and logistic regression. Institutional review board approval was obtained.

Results: In 235 patients identified, 28.9\% developed cardiotoxicity, of which $27.2 \%$ were acute, while chronic cardiotoxicity was observed in $8.9 \%$ of subjects. Patients who received optimal cardiac monitoring had a statistically significant higher odds of developing cardiotoxicities (odds ratio $=2.65$, confidence interval $=1.32-5.33$ ). The risk of cardiotoxicity was higher in subjects with a history of diabetes mellitus, those using daunorubicin, and concomitant filgrastim use. Adherence to guideline recommendations was only achieved in $25.1 \%$ of the population. Echocardiography was the most common monitoring method used. Conclusion: In this study, there was a high incidence of anthracyclines cardiotoxicity and poor compliance with cardiac monitoring recommendations for cancer patients on anthracyclines, which underscores acute and chronic cardiotoxicity in this population.

Keywords: anthracyclines, anthracyclines-induced cardiotoxicity, cardiac monitoring, cancer

\section{Background}

In the last decade, the treatment of many solid and haematological malignancies has advanced significantly. Anthracyclines, such as doxorubicin, epirubicin, and daunorubicin, are commonly used to treat various cancer types. ${ }^{1}$ Despite being highly effective, nevertheless, these agents come with a wide range of side effects, specifically cardiovascular toxicity of which some might be permanent. $^{2-4}$ The generation of free radicals with anthracyclines use may elevate cardiac biomarkers and decrease left ventricular ejection fraction. ${ }^{5,6}$ In the early stages of the left ventricle dysfunction, patients may not have any symptoms. However, it can
Correspondence: Hadeel Alkofide

Department of Clinical Pharmacy, College of Pharmacy, King Saud University, Riyadh,

II I49, Saudi Arabia

Tel +966503225I2I

Email halkofide@ksu.edu.sa 
progress to dilated cardiomyopathy and congestive heart failure. ${ }^{5,6}$ This problem influences the patient's quality of life, and it could be life-threatening and irreversible. ${ }^{7,8}$

Acute anthracyclines cardiotoxicity is identified during drug administration or soon after and presents mainly as transient arrhythmia, ST/T changes in electrocardiogram (ECG), with a pericarditis-myocarditis syndrome or decompensated acute cardiac failure. ${ }^{9}$ These acute effects are usually self-terminating by supportive therapy. ${ }^{9}$ On the other hand, chronic anthracyclines cardiotoxicity can occur within a year of therapy or several years after and usually is irreversible and lifelong. ${ }^{10}$ Chronic toxicity mainly consists of congestive heart failure with reduced ejection fraction. ${ }^{11}$ Studies suggest that subjects with certain risk factors are more susceptible to anthracyclines-induced cardiotoxicity. ${ }^{12-15}$ One of the main risk factors is the cumulative dose of anthracyclines, especially doxorubicin, in doses more than $400 \mathrm{mg} /$ $\mathrm{m} 2{ }^{12,13}$ Other risk factors include age, smoking, obesity (ie, body mass index above 30), and the presence of cardiovascular diseases. ${ }^{14,15}$ Previous exposure to cardiotoxic agents or coadministration of chemotherapy that have cardiac adverse effects can also increase the risk of anthracycline-induced cardiotoxicity. ${ }^{3,16}$ Most studies on anthracyclines safety report on chronic cardiotoxicity only and little are known on acute cardiotoxicity incidence. $^{10}$

Practice guidelines recommend periodic monitoring for cardiac function even before the first dose of anthracyclines is administered. ${ }^{17,18}$ Early detection is more helpful in both treating and preventing cardiotoxicity from progressing. ${ }^{19}$ There is little information on cardiac monitoring patterns in subjects receiving anthracyclines, and most available data is focused on subjects with breast cancer, and with the use of trastuzumab. ${ }^{20,21}$ Henry et al observed that the rate of chemotherapy-related cardiotoxicity in patients who received anthracycline-based regimens was more likely in those treated with both anthracyclines and trastuzumab. ${ }^{20}$ The authors also found that the use of anthracyclines was associated with higher odds of receiving cardiac monitoring adherence to guideline recommendations. ${ }^{20}$ One study in subjects with patients with lymphoma receiving anthracycline-based chemotherapy found that only one-third of patients received guideline-recommended cardiac monitoring. ${ }^{22}$

Given the limited data on acute and chronic anthracycline cardiotoxicity, and on adherence rates to cardiac monitoring, globally and specifically in the region, this cohort study aimed to estimate the incidence and determinants of anthracycline-induced cardiotoxicity, both acute and chronic. Also, we determined the rate of adherence to cardiac monitoring recommendations among anthracycline-treated patients and investigated whether this monitoring was associated with the incidence of cardiotoxicity.

\section{Method}

\section{Study Design and Setting}

This retrospective cohort study was performed at King Saud University Medical City (KSUMC), a tertiary-care teaching hospital, located in Riyadh, Saudi Arabia. Data were collected from medical records starting from June 2015 until May 2018.

\section{Participants}

Cancer patients 18 years of age or older were eligible if they received anthracyclines regardless of the type of cancer or malignancy. Patients who already have any cardiovascular dysfunction before the initiation of anthracyclines were excluded. Patients were followed from their cancer diagnosis date until they either died or no longer followed up at KSUMC. The last follow-up date was May 31, 2018. The study was approved by the institutional review board of King Saud University (Institutional review board number E-18-3127).

\section{Data Collection}

Demographic characteristics (eg, age, gender, and body mass index [BMI]), clinical (eg, type and stage of cancer and comorbidities), and treatment information (eg, type and doses of anthracycline, use of other medications, and use of radiation therapy) were collected for each eligible subject from the electronic medical record. Also, data were collected on monitoring parameters: echocardiography, radionuclide ventriculography (multiple-gated acquisition [MUGA] scans), ECG, serum cardiac biomarkers (troponin and natriuretic peptides), as well as cardiac magnetic resonance imaging (MRI). Date of medication use and the date of performing any of the monitoring parameters available in the patient electronic medical records were collected.

\section{Definitions}

Cardiotoxicity

Patients were categorized according to the presence of cardiotoxicity and the adherence guidelines monitoring 
recommendations. Cardiotoxicity was defined as a combination of either acute or chronic cardiotoxicity. Acute cardiotoxicity was defined as new ECG changes occurring after a single dose or a single course, with symptoms within 14 days from the end of treatment. Chronic cardiotoxicity was defined as the incidence of heart failure, either symptomatic or asymptomatic, as reported in medical records at any time after treatment. If left ventricular ejection fraction was reported in the medical records in subjects who developed heart failure that was noted as well. This definition was adapted from that used by others to define cardiac dysfunction associated with cancer therapy. ${ }^{20}$

\section{Adherence to Cardiac Monitoring}

Adherence to cardiac monitoring was based on the American Society of Clinical Oncology (ASCO) clinical practice guidelines recommendations. ${ }^{18}$ This was defined as a baseline cardiac evaluation before the first dose of anthracyclines and a subsequent follow-up cardiac evaluation during therapy. Baseline cardiac monitoring was defined as a test before any anthracycline dose; followup cardiac monitoring was defined as monitoring four months after initiating treatment. Methods for cardiac monitoring included either one or more of the following tests: echocardiograms, ECG, MUGA scans, and cardiac biomarkers. We also collected information on cardiacMRI.

\section{High-Risk for Cardiotoxicity}

According to the American Society of Clinical Oncology Clinical Practice Guidelines definition for high-risk patients, we identified subjects at high risk of cardiovascular toxicity. ${ }^{18}$ This criterion includes subjects using high-dose anthracycline (eg, doxorubicin $\geq 250 \mathrm{mg} / \mathrm{m} 2$, epirubicin $\geq 600 \mathrm{mg} / \mathrm{m} 2$ ). The high-risk criteria also included those receiving lower-dose anthracycline (eg, doxorubicin $<250 \mathrm{mg} / \mathrm{m} 2$, epirubicin $<600 \mathrm{mg} / \mathrm{m} 2$ ) and the presence of multiple cardiovascular risk factors $(\geq$ two risk factors), including smoking, hypertension, diabetes, dyslipidaemia, and obesity, during or after completion of therapy. Those receiving lower-dose anthracycline and older than 60 years of age at cancer treatment were also considered at high risk of cardiotoxicity. Finally, the high-risk definition also included those subjects on lowerdose anthracycline followed by trastuzumab (sequential therapy). The guideline also defines those subjects with compromised cardiac function, such as borderline low left ventricular ejection fraction and history of myocardial infarction and receiving lower-dose anthracycline were deemed as high-risk subjects. ${ }^{18}$ However, since we did not include subjects the pre-existing cardiovascular diseases this did not apply to this study population. In addition, the use of high dose radiotherapy ( $\geq 30$ grays) where the heart is in the treatment field or the use of lower-dose anthracycline in combination with lower-dose radiotherapy ( $<30$ grays) where the heart is in the treatment field puts subjects at high risk of cardiotoxicity, according to the guidelines. ${ }^{18}$ Nevertheless, such information on the treatment field for radiotherapy was not available. Therefore, this variable was used in the definition of high risk.

\section{Statistical Analysis}

Descriptive statistics were performed to summarize data. Data are shown as a summary for all subjects, and separately for those who developed cardiotoxicity, and also in whom guidelines were followed versus those who did not receive appropriate guideline recommendations. A Chisquare test was used to compare patient characteristics between groups. Univariable and multivariable logistic regressions were used to compare the outcome of having cardiotoxicity and the predictor of following treatment guidelines. Variables associated with cardiotoxicity in univariable models at $p$-values less than 0.1 were included in the multivariable model. As a sensitivity analysis, we ran the same regression models, including only subjects at high risk of cardiotoxicity. Logistic regression analysis was also performed to identify possible predictors for adherence to monitoring guidelines. Data were analyzed using $\mathrm{R}$ statistical program version 4.0.3. All tests are twotailed, and a p-value of $<0.05$ was considered significant.

\section{Results}

\section{Study Population}

A total of 235 patients were included in this study. Subjects were followed up for an average of 1.1 years and a standard deviation of $( \pm 0.9)$ years. The majority were males $(54.9 \%)$, and $74.5 \%$ (175/235) were below the age of 60 years. Sixty percent of participants had a BMI of more than $30 \mathrm{~kg} / \mathrm{m} 2$. Most study participants were considered at high risk of cardiotoxicity $(88.9 \%)$. Lymphoma was the most common type of cancer in the study population $(56.2 \%)$, and the cancer was of new diagnosis in most patients $(91.5 \%)$. Doxorubicin was the most commonly used anthracycline and most subjects on 
high-dose anthracyclines. About half of the subjects received biological therapy, mainly using rituximab, cyclophosphamide, and mesna. Details of the study participants are presented in Table 1.

\section{Cardiotoxicity}

In this cohort, $28.9 \%$ of subjects developed cardiotoxicity, which included both acute and chronic toxicities. The incidence of chronic (ie, delayed) cardiotoxicity was $8.9 \%$, while acute cardiotoxicity was $27.2 \%$. Subjects who developed cardiotoxicity were more likely to be diabetic $(p=0.033)$. The type of cancer was also different between those who developed and did not develop cardiotoxicity $(p=0.001)$. There was a significant difference in the anthracycline agents used between those with and without cardiotoxicity $(\mathrm{p}<0.001)$. Adherence to monitoring recommendations was higher in subjects who developed cardiotoxicity $(p=0.014)$. Other clinical characteristics were similar primarily across the groups. These characteristics are summarized in Table 1. Detailed types of cardiotoxicities observed are presented in Supplementary Table S1.

Univariable and multivariable logistic regression models on the outcome of cardiotoxicity are available in Table 2. After adjusting for possible confounders, the odds ratio for developing cardiotoxicity was 2.73 in subjects who were diabetic versus non-diabetics. The use of daunorubicin was associated with higher odds of cardiotoxicity compared to doxorubicin in multivariable analysis. Also, cardiotoxicity was significantly higher with the concomitant use of filgrastim with anthracyclines. Furthermore, optimal monitoring was associated with higher odds of cardiotoxicity (Table 2). Sensitivity analysis using only subjects at high risk of cardiotoxicity $(n=209)$, the results remained the same, except that the use of filgrastim was no longer associated with higher odds of cardiotoxicity (Supplementary Table S2).

\section{Cardiac Monitoring}

Only $25.1 \%$ of the study participants received optimal monitoring for cardiotoxicity. Cardiac monitoring was more frequent in subjects who developed cardiotoxicity $(36.8 \%)$ than those who did not develop any toxicities (20.4\%). Subjects who developed cardiotoxicity were more likely to have monitoring tests performed during therapy $(57.4 \%$ in subjects with cardiotoxicity versus $26.3 \%$ in those without toxicities). The most common test performed at baseline was echocardiography
(29.8\%), followed by ECG (22.1\%). On the other hand, at follow-up testing, the most common test performed were cardiac biomarkers $(20.9 \%)$, followed by ECG (18.3\%). Here, MRI testing was only performed on two study subjects during the study period. These results are presented in Table 3.

Study participants' characteristics across those who received optimal cardiac monitoring versus those who received suboptimal monitoring are available in Table 4. There were generally no differences between the groups in terms of clinical and demographic characteristics. However, those who received optimal cardiac monitoring were more likely to be active smokers $(16.9 \%)$ than those who did not receive optimal cardiac monitoring (6.2\%). Cardiotoxicity was more prevalent in those who received optimal cardiac monitoring $(42.4 \%)$ versus those who did not receive any monitoring $(24.4 \%)$. We attempted to perform logistic regression analysis on possible predictors for optimal adherence to cardiac monitoring recommendations. However, since only smoking was significantly correlated with cardiac monitoring, multiple logistic regression analysis was not performed. Results of univariable regression analyses are presented in Supplementary Table S3. Although we tried collecting left ventricular ejection fraction information on all study participants, these data were only available on a subset of subjects $(n=30)$. In those participants, the average and standard deviation of the left ventricular ejection fraction were $64.8 \pm 6.4$ and $56.5 \pm 10.7 \%$ before and after treatment initiation, respectively.

\section{Discussion}

This study found a generally high incidence rate of cardiotoxicity following anthracycline use in subjects with cancer. However, this incidence rate was mostly driven by acute toxicity. There are limited reports on the incidence of acute anthracycline toxicity. ${ }^{10}$ Nevertheless, in this cohort, we did observe a high incidence of ECG changes shortly after the initiation of anthracyclines. The rate of chronic or delayed toxicity here was similar to that reported by other studies. ${ }^{12,19}$ In a prospective study on cancer patients receiving anthracyclines, the overall incidence of cardiotoxicity was $9 \% .{ }^{19}$ Another retrospective cohort study on patients with lymphoma compared the incidence of congestive heart failure in patients with anthracycline regimen to non-anthracycline regimen found that $9.4 \%$ of patient treated with anthracycline developed heart failure after 
Table I Characteristics of Study Participants Based on Incidence of Cardiotoxicity

\begin{tabular}{|c|c|c|c|c|}
\hline & Overall & Cardiotoxicity & No-Cardiotoxicity & p-value* \\
\hline & $(\mathrm{N}=235)$ & $(\mathrm{N}=68)$ & $(N=167)$ & \\
\hline Gender & & & & 0.228 \\
\hline Male & $129(54.9 \%)$ & $42(61.8 \%)$ & $87(52.1 \%)$ & \\
\hline Female & $106(45.1 \%)$ & $26(38.2 \%)$ & $80(47.9 \%)$ & \\
\hline Age & & & & 0.481 \\
\hline$<60$ years & 175 (74.5\%) & $48(70.6 \%)$ & $127(76.0 \%)$ & \\
\hline$>60$ years & $60(25.5 \%)$ & 20 (29.4\%) & $40(24.0 \%)$ & \\
\hline BMI > 30 & $|4|(60.0 \%)$ & $40(58.8 \%)$ & I0I (60.5\%) & 0.930 \\
\hline Current Smoking & 21 (8.9\%) & $5(7.4 \%)$ & $16(9.6 \%)$ & 0.771 \\
\hline History of Hypertension & $66(28.1 \%)$ & $24(35.3 \%)$ & $42(25.1 \%)$ & 0.159 \\
\hline History of Dyslipidaemia & $38(16.2 \%)$ & $15(22.1 \%)$ & $23(13.8 \%)$ & 0.171 \\
\hline History Diabetes & 44 (18.7\%) & $19(27.9 \%)$ & 25 (15.0\%) & 0.033 \\
\hline High-Risk for Cardiotoxicity & 209 (88.9\%) & 61 (89.7\%) & 148 (88.6\%) & 0.991 \\
\hline High dose anthracycline & $155(66.0 \%)$ & $4 \mathrm{I}(60.3 \%)$ & $114(68.3 \%)$ & 0.309 \\
\hline Low dose anthracycline and age above 60 years old & $26(11.1 \%)$ & $18(10.8 \%)$ & $8(11.8 \%)$ & 0.991 \\
\hline Low dose anthracycline with $\geq$ two cardiovascular risk factors & $18(7.7 \%)$ & $9(5.4 \%)$ & $9(13.2 \%)$ & 0.080 \\
\hline Low dose anthracycline followed by trastuzumab & $10(4.3 \%)$ & $3(4.4 \%)$ & $7(4.2 \%)$ & 0.990 \\
\hline Type of Cancer & & & & 0.001 \\
\hline Breast & $23(9.8 \%)$ & $5(7.4 \%)$ & $18(10.8 \%)$ & \\
\hline Lymphoma & $132(56.2 \%)$ & $29(42.6 \%)$ & $103(61.7 \%)$ & \\
\hline Leukaemia & $32(13.6 \%)$ & 19 (27.9\%) & $13(7.8 \%)$ & \\
\hline Hepatocellular carcinoma & $12(5.1 \%)$ & $2(2.9 \%)$ & $10(6.0 \%)$ & \\
\hline Others & $36(15.3 \%)$ & $13(19.1 \%)$ & $23(13.8 \%)$ & \\
\hline Cancer Status & & & & 0.234 \\
\hline New & $215(91.5 \%)$ & $59(86.8 \%)$ & I56 (93.4\%) & \\
\hline Recurrent & $12(5.1 \%)$ & $5(7.4 \%)$ & 7 (4.2\%) & \\
\hline Relapse & $8(3.4 \%)$ & $4(5.9 \%)$ & $4(2.4 \%)$ & \\
\hline Anthracycline type & & & & $<0.001$ \\
\hline Doxorubcin & $193(82.1 \%)$ & $43(63.2 \%)$ & $150(89.8 \%)$ & \\
\hline Liposomal doxorubcin & $15(6.4 \%)$ & $8(11.8 \%)$ & 7 (4.2\%) & \\
\hline Danurubicin & $27(11.5 \%)$ & $17(25.0 \%)$ & $10(6.0 \%)$ & \\
\hline Route of Administration & & & & $0.774 I$ \\
\hline Continuous infusion & 90 (38.3\%) & 27 (39.7\%) & $63(37.7 \%)$ & \\
\hline Bolus & 75 (31.9\%) & $23(33.8 \%)$ & $52(31.1 \%)$ & \\
\hline Both & $70(29.8 \%)$ & $18(26.5 \%)$ & $52(31.1 \%)$ & \\
\hline Radiotherapy & $167(71.1 \%)$ & 53 (77.9\%) & 114 (68.3\%) & 0.185 \\
\hline Biologic Therapy & & & & 0.743 \\
\hline Rituximab & $109(46.4 \%)$ & $32(47.1 \%)$ & 77 (46.1\%) & \\
\hline Trastuzumab & $10(4.3 \%)$ & $3(4.4 \%)$ & 7 (4.2\%) & \\
\hline Others & $9(3.8 \%)$ & $4(5.9 \%)$ & $5(3.0 \%)$ & \\
\hline Not on biologics & $107(45.5 \%)$ & $29(42.6 \%)$ & $78(46.7 \%)$ & \\
\hline Use of Cyclophosamide & $125(53.2 \%)$ & $37(54.4 \%)$ & $88(52.7 \%)$ & 0.924 \\
\hline
\end{tabular}

(Continued) 
Table I (Continued).

\begin{tabular}{|l|c|c|c|c|}
\hline & Overall & Cardiotoxicity & No-Cardiotoxicity & P-value* \\
\cline { 2 - 5 } & $\mathbf{( N = 2 3 5 )}$ & $\mathbf{( N = 6 8 )}$ & $\mathbf{( N = 1 6 7 )}$ & $110(65.9 \%)$ \\
\hline Use of Vincristin & $161(68.5 \%)$ & $51(75.0 \%)$ & 0.226 \\
\hline Use of Mesna & $122(51.9 \%)$ & $40(58.8 \%)$ & $82(49.1 \%)$ & 0.227 \\
\hline Use of Filgrastim & $99(42.1 \%)$ & $35(51.5 \%)$ & $64(38.3 \%)$ & 0.088 \\
\hline
\end{tabular}

Notes: *Chi-square test was used to compare categorical data. Data presented as number (percent).

Abbreviation: BMI, body mass index.

9-years follow-up compared to $0.8 \%$ of patients treated with a non-anthracycline regimen. ${ }^{12}$

Previous studies found a relationship between the cumulative anthracyclines dose, patients age, and the use of radiotherapy with the development of cardiotoxicity. ${ }^{12-}$ ${ }^{15,19-21}$ Here we were unable to demonstrate such associations. This could be explained by the fact that almost $90 \%$ of study participants were considered at high risk of cardiotoxicity. This indicates being on either high dose anthracyclines, having pre-existing cardiovascular conditions, and above the age of 60 , among other risk factors identified here. Interestingly, in a study by Cardinale et al, although they did not include subjects with pre-existing cardiovascular disease, similar to the study here, there was a low number of cardiotoxicities observed. ${ }^{23}$ This could be explained by the fact that in this cohort there was a high rate of cardiovascular disease risk factors compared to the study by Cardinale et al. ${ }^{23}$ For instance, here $28 \%$ of the study subjects had hypertension, and about $19 \%$ had diabetes, while in the other study only $3 \%$ and $4 \%$ of the participants had hypertension or diabetes, respectively. ${ }^{23}$ In this cohort, concomitant use of filgrastim was associated with double the odds of developing cardiotoxicity compared to no concomitant use of filgrastim. This could

Table 2 Association Between Cardiotoxicity and Clinical Characteristics Using Logistic Regression Model

\begin{tabular}{|c|c|c|c|c|c|c|}
\hline & \multicolumn{3}{|c|}{$\begin{array}{c}\text { Univariable Regression Analysis for } \\
\text { Cardiotoxicity }\end{array}$} & \multicolumn{3}{|c|}{$\begin{array}{l}\text { Multivariable Regression Analysis for } \\
\text { Cardiotoxicity }\end{array}$} \\
\hline & OR & $95 \% \mathrm{Cl}$ & p-value & OR & $95 \% \mathrm{Cl}$ & p-value \\
\hline History Diabetes & 2.20 & I.II, 4.34 & 0.02 & 2.73 & $1.27,5.88$ & 0.01 \\
\hline \multicolumn{7}{|l|}{ Type of cancer } \\
\hline Breast & Ref & & $<0.001$ & Ref & & \\
\hline Lymphoma & 1.01 & $0.37,3.28$ & & 1.20 & $0.4 I, 4.13$ & 0.80 \\
\hline Leukemia & 5.26 & $1.64,19.30$ & & 2.83 & $0.59,14.10$ & 0.20 \\
\hline Hepatocellular carcinoma & 0.72 & $0.09,4.06$ & & 1.22 & $0.14,7.97$ & 0.80 \\
\hline Others & 2.03 & $0.64,7.31$ & & 1.87 & $0.47,7.83$ & 0.40 \\
\hline Athracycline Agent & & & $<0.001$ & & & \\
\hline Doxorubcin & Ref & & & Ref & & \\
\hline Liposomal doxorubcin & 3.99 & $1.36,12.00$ & & 2.51 & $0.66,10.10$ & 0.20 \\
\hline Danurubicin & 5.93 & $2.57,14.40$ & & 4.51 & $1.16,17.90$ & 0.03 \\
\hline \multicolumn{7}{|l|}{ Supportive therapy } \\
\hline Filgrastim & 1.71 & $0.97,3.02$ & 0.07 & 2.37 & $1.23,4.71$ & 0.01 \\
\hline \multicolumn{7}{|l|}{ Outcome } \\
\hline Adherence to cardiac monitoring & 2.27 & $1.22,4.23$ & 0.01 & 2.65 & $1.32,5.33$ & 0.01 \\
\hline
\end{tabular}

Abbreviations: $\mathrm{Cl}$, confidence interval; $\mathrm{OR}$, odds ratio. 
Table 3 Cardiac Monitoring Based on Incidence of Cardiotoxicity

\begin{tabular}{|c|c|c|c|c|}
\hline & Overall & Cardiotoxicity & No-Cardiotoxicity & p-value* \\
\hline & $(\mathbf{N}=\mathbf{2 3 5})$ & $(N=68)$ & $(N=167)$ & \\
\hline Optimal Adherence to Cardiac Monitoring & $59(25.1 \%)$ & $25(36.8 \%)$ & $34(20.4 \%)$ & 0.014 \\
\hline \multicolumn{5}{|l|}{ Monitoring parameters } \\
\hline Any test at baseline & $125(53.2 \%)$ & $43(63.2 \%)$ & $82(49.1 \%)$ & 0.07 \\
\hline \multicolumn{5}{|l|}{ Type of Baseline Test } \\
\hline Echocardiography & $70(29.8 \%)$ & $27(39.7 \%)$ & $43(25.7 \%)$ & 0.05 \\
\hline MUGA scan & $48(20.4 \%)$ & $13(19.1 \%)$ & $35(21.0 \%)$ & 0.89 \\
\hline ECG & $52(22.1 \%)$ & $20(29.4 \%)$ & $32(19.2 \%)$ & 0.12 \\
\hline Cardiac biomarkers & $43(18.3 \%)$ & $17(25.0 \%)$ & $26(15.6 \%)$ & 0.19 \\
\hline Any test at follow-up & $83(35.3 \%)$ & 39 (57.4\%) & $44(26.3 \%)$ & $<0.001$ \\
\hline \multicolumn{5}{|l|}{ Type of Follow-Up Test } \\
\hline Echocardiography & $28(11.9 \%)$ & $10(14.7 \%)$ & $18(10.8 \%)$ & 0.54 \\
\hline MUGA scan & 19 (8.1\%) & 7 (10.3\%) & 12 (7.2\%) & 0.59 \\
\hline ECG & $43(18.3 \%)$ & $26(38.2 \%)$ & $17(10.2 \%)$ & $<0.001$ \\
\hline Cardiac biomarkers & 49 (20.9\%) & 21 (30.9\%) & $28(16.8 \%)$ & 0.03 \\
\hline \multicolumn{5}{|l|}{ Other tests } \\
\hline MRI & $2(0.9 \%)$ & $2(2.9 \%)$ & $0(0.0 \%)$ & 0.149 \\
\hline
\end{tabular}

Notes: *Chi-square test was used to compare categorical data. Data presented as number (percent).

Abbreviations: ECG, electrocardiogram; MRI, magnetic resonance imaging; MUGA, multiple-gated acquisition.

be explained by the fact that these subjects who require the use of filgrastim are more likely to develop adverse events from anthracyclines, whether these adverse events were cardiotoxicity or neutropenia.

Here, there was an association between having diabetes and the development of cardiotoxicity, similar to that reported in previous cohorts. ${ }^{15}$ Looking at each anthracycline separately, we observe the highest incidence rate of cardiotoxicity with daunorubicin. However, this association was no longer significant in multivariable regression analysis. There were no differences observed between daunorubicin and idarubicin in terms of cardiotoxicity in a systematic review and meta-analysis. ${ }^{24}$

This study aimed to identify cardiac monitoring parameters performed on patients using anthracycline and compare them with international guideline recommendations. We did observe suboptimal adherence to cardiac monitoring, as only a quarter of subjects received the required monitoring for cardiotoxicity. There are limited data regarding cardiac monitoring in subjects receiving anthracyclines, and most studies focus on cardiac monitoring associated with trastuzumab use, which is reported to be suboptimal. ${ }^{20}$ Patients on anthracycline should be monitored closely, even before starting treatment, as early detection helps to treat and prevent cardiotoxicity. ${ }^{19}$

Subjects who developed cardiotoxicity were more likely to receive optimal guideline adherence to cardiac monitoring. However, a higher incidence of cardiotoxicity is expected in those who received optimal monitoring, and the true rate of cardiotoxicity might have been underestimated. Studies show that monitoring patients on anthracyclines will help in the identification of cardiotoxicity. ${ }^{19}$ Cardiac monitoring is usually performed with an echocardiogram or with MUGA scans. ${ }^{25}$ The following parameters are also recommended for monitoring such as serum natriuretic peptides and cardiac troponins. ${ }^{17,18,26}$ For high-risk patients, routine monitoring with an echocardiogram is recommended during treatment. ${ }^{18}$ In this study, the most common tests used before the initiation of anthracyclines were echocardiogram and ECG. While during therapy, the most common test performed were cardiac biomarkers, followed by ECG. 
Table 4 Characteristics of Study Participants Based on Adherence to Cardiac Monitoring

\begin{tabular}{|c|c|c|c|c|}
\hline & Overall & Optimal Monitoring & Suboptimal Monitoring & p-value* \\
\hline & $(\mathrm{N}=\mathbf{2 3 5})$ & $(N=59)$ & $(N=176)$ & \\
\hline Gender & & & & 0.21 \\
\hline Male & $129(54.9 \%)$ & $37(62.7 \%)$ & 92 (52.3\%) & \\
\hline Female & $106(45.1 \%)$ & $22(37.3 \%)$ & $84(47.7 \%)$ & \\
\hline Age & & & & 0.846 \\
\hline$<60$ years & $175(74.5 \%)$ & $45(76.3 \%)$ & $130(73.9 \%)$ & \\
\hline$>60$ years & $60(25.5 \%)$ & $14(23.7 \%)$ & $46(26.1 \%)$ & \\
\hline BMI > 30 & $|4|(60.0 \%)$ & $35(59.3 \%)$ & $106(60.2 \%)$ & 0.902 \\
\hline Active Smoking & $21(8.9 \%)$ & $10(16.9 \%)$ & II (6.2\%) & 0.026 \\
\hline History of Hypertension & $66(28.1 \%)$ & $15(25.4 \%)$ & $51(29.0 \%)$ & 0.720 \\
\hline History of Dyslipidaemia & $38(16.2 \%)$ & $9(15.3 \%)$ & $29(16.5 \%)$ & 0.987 \\
\hline History Diabetes & 44 (18.7\%) & $10(16.9 \%)$ & $34(19.3 \%)$ & 0.833 \\
\hline High-Risk for Cardiotoxicity & $209(88.9)$ & $52(88.1 \%)$ & 157 (89.2\%) & 0.990 \\
\hline High dose anthracycline & $155(66.0 \%)$ & $39(66.1 \%)$ & $116(65.9 \%)$ & 0.978 \\
\hline Low dose anthracycline and age above 60 years old & $26(11.1 \%)$ & $20(11.4 \%)$ & $6(10.2 \%)$ & 0.989 \\
\hline Low dose anthracycline with $\geq$ two cardiovascular risk factors & I8 (7.7\%) & II (6.2\%) & 7 ( $11.9 \%)$ & 0.263 \\
\hline Low dose anthracycline followed by trastuzumab & $10(4.3 \%)$ & $4(6.8 \%)$ & $6(3.4 \%)$ & 0.902 \\
\hline Type of cancer & & & & 0.442 \\
\hline Breast & $23(9.8 \%)$ & $8(13.6 \%)$ & $15(8.5 \%)$ & \\
\hline Lymphoma & $132(56.2 \%)$ & $34(57.6 \%)$ & $98(55.7 \%)$ & \\
\hline Leukaemia & $32(13.6 \%)$ & $9(15.3 \%)$ & $23(\mid 3.1 \%)$ & \\
\hline Hepatocellular carcinoma & $12(5.1 \%)$ & I (I.7\%) & II (6.2\%) & \\
\hline Others & $36(15.3 \%)$ & $7(11.9 \%)$ & $29(16.5 \%)$ & \\
\hline Cancer Status & & & & 0.704 \\
\hline New & $215(91.5 \%)$ & 55 (93.2\%) & $160(90.9 \%)$ & \\
\hline Recurrent & $12(5.1 \%)$ & $3(5.1 \%)$ & $9(5.1 \%)$ & \\
\hline Relapse & $8(3.4 \%)$ & I (I.7\%) & $7(4.0 \%)$ & \\
\hline Anthracycline type & & & & 0.738 \\
\hline Doxorubcin & $193(82.1 \%)$ & 47 (79.7\%) & 146 (83.0\%) & \\
\hline Liposomal doxorubcin & $15(6.4 \%)$ & $5(8.5 \%)$ & $10(5.7 \%)$ & \\
\hline Danurubicin & 27 (I I.5\%) & 7 (II.9\%) & $20(11.4 \%)$ & \\
\hline Route of Administration & & & & 0.512 \\
\hline Continuous infusion & $90(38.3 \%)$ & $20(33.9 \%)$ & $70(39.8 \%)$ & \\
\hline Bolus & 75 (31.9\%) & $18(30.5 \%)$ & $57(32.4 \%)$ & \\
\hline Both & 70 (29.8\%) & $21(35.6 \%)$ & 49 (27.8\%) & \\
\hline High Dose of Anthracyclines & $155(66.0 \%)$ & $39(66.1 \%)$ & 116 (65.9\%) & 0.978 \\
\hline Radiotherapy & $167(71.1 \%)$ & $42(71.2 \%)$ & 125 (7I.0\%) & 0.981 \\
\hline Biological Therapy & & & & 0.518 \\
\hline
\end{tabular}

(Continued) 
Table 4 (Continued).

\begin{tabular}{|c|c|c|c|c|}
\hline & Overall & Optimal Monitoring & Suboptimal Monitoring & p-value* \\
\hline & $(\mathrm{N}=\mathbf{2 3 5})$ & $(\mathrm{N}=59)$ & $(N=176)$ & \\
\hline Rituximab & $109(46.4 \%)$ & 26 (44.1\%) & 83 (47.2\%) & \\
\hline Trastuzumab & 10 (4.3\%) & $4(6.8 \%)$ & $6(3.4 \%)$ & \\
\hline Others & $9(3.8 \%)$ & I (I.7\%) & $8(4.5 \%)$ & \\
\hline Not on biologics & $107(45.5 \%)$ & 28 (47.5\%) & 79 (44.9\%) & \\
\hline Use of Cyclophosamide & $125(53.2 \%)$ & 31 (52.5\%) & 94 (53.4\%) & 0.908 \\
\hline Use of Vincristin & $16 \mid(68.5 \%)$ & 39 (66.1\%) & $122(69.3 \%)$ & 0.765 \\
\hline Use of Mesna & $122(51.9 \%)$ & $30(50.8 \%)$ & $92(52.3 \%)$ & 0.969 \\
\hline Use of Filgrastim & 99 (42.1\%) & $25(42.4 \%)$ & $74(42.0 \%)$ & 0.965 \\
\hline Cardiotoxicity & $68(28.9 \%)$ & 25 (42.4\%) & $43(24.4 \%)$ & \\
\hline
\end{tabular}

Notes: *Chi-square test was used to compare categorical data. Data presented as number (percent).

Abbreviation: BMI, body mass index.

There is no consensus in clinical practice guidelines on the preferred mentoring technique, although most recommend echocardiogram or MUGA scans. ${ }^{27}$ However, these tests might not be readily available for all patients; therefore, some recommend the use of cardiac biomarkers. ${ }^{6}$ One study showed that early changes in troponin levels in subjects using anthracyclines are associated with subsequent cardiotoxicity. ${ }^{6}$ The combination of multiple markers may have a common benefit in predicting subsequent cardiotoxicity. ${ }^{6}$

This study has several limitations. First, as a retrospective single-centre study, the sample size was small, with limited generalizability. Second, we did observe poor documentation of the results of cardiac monitoring in the medical records. Third, our patient population mainly had lymphoma. Therefore, the results of this study might not apply to other types of cancer. Fourth, we did not include information on the use of cardiovascular medications in this population, which might have impacted the development of cardiotoxicity. Fifth, baseline data on cardiac function tests, such as left ventricular ejection fraction was not available for all subjects, nevertheless, we did attempt to report the available information when feasible. Sixth, although we attempted to collect follow-up dates for study participants, we were unable to obtain the exact follow-up time for the acute and chronic cardiotoxicity. Lastly, the percentage of chronic cardiotoxicity was underestimated because of the short study period, loss of follow up, and inadequate cardiac monitoring.

\section{Conclusions}

To our knowledge, our study is the first of its kind to estimate cardiotoxicity rates and cardiac monitoring in cancer subjects in Saudi Arabia. We did observe a more significant incidence rate of acute cardiotoxicity. However, these results underestimate the true incidence of cardiotoxicity due to the suboptimal adherence to cardiac monitoring. The incidence rate of chronic cardiotoxicity was aligned with that seen in other populations. Future observational studies with prospective follow-up are required to estimate the true incidence of anthracycline-induced acute together with chronic cardiotoxicity.

\section{Abbreviations}

ASCO, American Society of Clinical Oncology; BMI, body mass index; ECG, electrocardiogram; KSUMC, King Saud University Medical City; MRI, magnetic resonance imaging; MUGA, multiple-gated acquisition.

\section{Data Sharing Statement}

The datasets used and analyzed during the current study are available from the corresponding author on reasonable request.

\section{Ethical Approval}

The study was approved by the institutional review board of King Saud University (Institutional review board number E-18-3127). All data were anonymized to maintain participant's privacy, and the study was conducted in accordance with the Declaration of Helsinki. In light of 
the retrospective and anonymous nature of the study, the Ethics Committee did not require written informed consent provided by participants.

\section{Acknowledgments}

We thank Undergraduate Research Support Program, for their support. This research project was supported by a grant from the "Research Center of the Center for Female Scientific and Medical Colleges", Deanship of Scientific Research, King Saud University. We would also like to thank Miss Rahaf Almusned and Miss Lujain Alnoghaimshi for their assistance in data collection.

\section{Author Contributions}

All authors made a significant contribution to the work reported, whether that is in the conception, study design, execution, acquisition of data, analysis and interpretation, or in all these areas; took part in drafting, revising or critically reviewing the article; gave final approval of the version to be published; have agreed on the journal to which the article has been submitted; and agree to be accountable for all aspects of the work.

\section{Funding}

This research project was supported by a grant from the "Research Center of the Center for Female Scientific and Medical Colleges", Deanship of Scientific Research, King Saud University.

\section{Disclosure}

The abstract of this paper was presented at the $9^{\text {th }}$ college of pharmacy research day (COPRD) at King Saud University, College of Pharmacy, as a presentation with interim findings. The authors report no conflicts of interest in this work.

\section{References}

1. Hortobágyi GN. Anthracyclines in the treatment of cancer. An overview. Drugs. 1997;54(Suppl 4):1-7. doi:10.2165/00003495199700544-00003

2. Smith LA, Cornelius VR, Plummer CJ, et al. Cardiotoxicity of anthracycline agents for the treatment of cancer: systematic review and meta-analysis of randomised controlled trials. BMC Cancer. 2010;10:337. doi:10.1186/1471-2407-10-337

3. Livshits Z, Rao RB, Smith SW. An approach to chemotherapy-associated toxicity. Emerg Med Clin North Am. 2014;32(1):167-203. doi:10.1016/j.emc.2013.09.002

4. Leong SL, Chaiyakunapruk N, Lee SWH. Antineoplastic-related cardiovascular toxicity: a systematic review and meta-analysis in Asia. Crit Rev Oncol Hematol. 2019;141:95-101. doi:10.1016/j. critrevonc.2019.05.017
5. Simůnek T, Stérba M, Popelová O, Adamcová M, Hrdina R, Gersl V. Anthracycline-induced cardiotoxicity: overview of studies examining the roles of oxidative stress and free cellular iron. Pharmacol Rep. 2009;61(1):154-171. doi:10.1016/S1734-1140(09)70018-0

6. Ky B, Putt M, Sawaya H, et al. Early increases in multiple biomarkers predict subsequent cardiotoxicity in patients with breast cancer treated with doxorubicin, taxanes, and trastuzumab [published correction appears in J Am Coll Cardiol. 2016 Mar 22;67(11):1385]. J Am Coll Cardiol. 2014;63(8):809-816. doi:10.1016/j.jacc.2013.10.061

7. Lotrionte M, Biondi-Zoccai G, Abbate A, et al. Review and meta-analysis of incidence and clinical predictors of anthracycline cardiotoxicity. Am J Cardiol. 2013;112(12):1980-1984. doi:10.1016/ j.amjcard.2013.08.026

8. Armstrong GT, Liu Q, Yasui Y, et al. Late mortality among 5-year survivors of childhood cancer: a summary from the Childhood Cancer Survivor Study. J Clin Oncol. 2009;27(14):2328-2338. doi:10.1200/JCO.2008.21.1425

9. Dazzi H, Kaufmann K, Follath F. Anthracycline-induced acute cardiotoxicity in adults treated for leukaemia. Analysis of the clinicopathological aspects of documented acute anthracycline-induced cardiotoxicity in patients treated for acute leukaemia at the University Hospital of Zürich, Switzerland, between 1990 and 1996. Ann Oncol. 2001;12(7):963-966. doi:10.1023/a:1011196910325

10. Volkova M, Russell R 3rd. Anthracycline cardiotoxicity: prevalence, pathogenesis and treatment. Curr Cardiol Rev. 2011;7(4):214-220. doi: $10.2174 / 157340311799960645$

11. Eschenhagen T, Force T, Ewer MS, et al. Cardiovascular side effects of cancer therapies: a position statement from the Heart Failure Association of the European Society of Cardiology. Eur J Heart Fail. 2011;13(1):1-10. doi:10.1093/eurjhf/hfq213

12. Baech J, Hansen SM, Lund PE, et al. Cumulative anthracycline exposure and risk of cardiotoxicity; a Danish nationwide cohort study of 2440 lymphoma patients treated with or without anthracyclines [published correction appears in $\mathrm{Br} \mathrm{J}$ Haematol. 2020 Mar;188(5):801]. $\quad \mathrm{Br} \quad J \quad$ Haematol. 2018;183(5):717-726. doi:10.1111/bjh.15603

13. Skubitz KM, Blaes AH, Konety SH, Francis GS. Cardiac safety profile of patients receiving high cumulative doses of pegylated-liposomal doxorubicin: use of left ventricular ejection fraction is of unproven value. Cancer Chemother Pharmacol. 2017;80 (4):787-798. doi:10.1007/s00280-017-3420-8

14. Reinbolt RE, Patel R, Pan X, et al. Risk factors for anthracycline-associated cardiotoxicity. Support Care Cancer. 2016;24(5):2173-2180. doi:10.1007/s00520-015-3008-y

15. Guenancia C, Lefebvre A, Cardinale D, et al. Obesity as a risk factor for anthracyclines and trastuzumab cardiotoxicity in breast cancer: a systematic review and meta-analysis. J Clin Oncol. 2016;34 (26):3157-3165. doi:10.1200/JCO.2016.67.4846

16. Hequet O, Le QH, Moullet I, et al. Subclinical late cardiomyopathy after doxorubicin therapy for lymphoma in adults. J Clin Oncol. 2004;22(10):1864-1871. doi:10.1200/JCO.2004.06.033

17. Zamorano JL, Lancellotti P, Rodriguez Muñoz D, et al. 2016 ESC position paper on cancer treatments and cardiovascular toxicity developed under the auspices of the ESC Committee for Practice Guidelines: the Task Force for cancer treatments and cardiovascular toxicity of the European Society of Cardiology (ESC) [published correction appears in Eur Heart J. 2016 Dec 24]. Eur Heart J. 2016;37(36):2768-2801. doi:10.1093/eurheartj/ehw211

18. Armenian SH, Lacchetti C, Barac A, et al. Prevention and monitoring of cardiac dysfunction in survivors of adult cancers: American Society of Clinical Oncology Clinical Practice Guideline. J Clin Oncol. 2017;35(8):893-911. doi:10.1200/JCO.2016.70.5400

19. Cardinale D, Colombo A, Bacchiani G, et al. Early detection of anthracycline cardiotoxicity and improvement with heart failure therapy. Circulation. 2015;131(22):1981-1988. doi:10.1161/ CIRCULATIONAHA.114.013777 
20. Henry ML, Niu J, Zhang N, Giordano SH, Chavez-MacGregor M Cardiotoxicity and cardiac monitoring among chemotherapy-treated breast cancer patients. JACC Cardiovasc Imaging. 2018;11 (8):1084-1093. doi:10.1016/j.jcmg.2018.06.005

21. Chavez-MacGregor M, Niu J, Zhang N, et al. Cardiac monitoring during adjuvant trastuzumab-based chemotherapy among older patients with breast cancer. J Clin Oncol. 2015;33(19):2176-2183. doi:10.1200/JCO.2014.58.9465

22. Hung OY, Brown JR, Dai T, Easley KA, Flowers CR, Parashar S. Pattern of cardiac surveillance among patients with lymphoma receiving anthracycline-based chemotherapy. BMJ Open. 2015;5 (10):e008350. doi:10.1136/bmjopen-2015-008350

23. Cardinale D, Ciceri F, Latini R, et al. Anthracycline-induced cardiotoxicity: a multicenter randomised trial comparing two strategies for guiding prevention with enalapril: the International CardioOncology Society-one trial. Eur J Cancer. 2018;94:126-137. doi:10.1016/j. ejca.2018.02.005
24. Owattanapanich W, Owattanapanich N, Kungwankiattichai S, Ungprasert P, Ruchutrakool T. Efficacy and toxicity of idarubicin versus high-dose daunorubicin for induction chemotherapy in adult acute myeloid leukemia: a systematic review and meta-analysis. Clin Lymphoma Myeloma Leuk. 2018;18(12):814-821.e3. doi:10.1016/j. clm1.2018.08.008

25. Curigliano G, Cardinale D, Dent S, et al. Cardiotoxicity of anticancer treatments: epidemiology, detection, and management. CA Cancer J Clin. 2016;66(4):309-325. doi:10.3322/caac.21341

26. Cardinale D, Iacopo F, Cipolla CM. Cardiotoxicity of anthracyclines. Front Cardiovasc Med. 2020;7:26. doi:10.3389/fcvm.2020.00026

27. Henriksen PA. Anthracycline cardiotoxicity: an update on mechanisms, monitoring and prevention. Heart. 2018;104(12):971-977. doi:10.1136/heartjnl-2017-312103

\section{Publish your work in this journal}

Cancer Management and Research is an international, peer-reviewed open access journal focusing on cancer research and the optimal use of preventative and integrated treatment interventions to achieve improved outcomes, enhanced survival and quality of life for the cancer patient.
The manuscript management system is completely online and includes a very quick and fair peer-review system, which is all easy to use. Visit http://www.dovepress.com/testimonials.php to read real quotes from published authors. 